Supporting information

\title{
Highly-Flexible Self-Powered Organolead Trihalide Perovskite Photodetectors with Gold Nanowire Networks as Transparent Electrodes
}

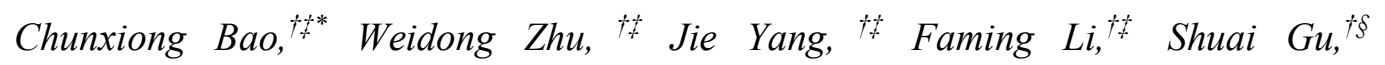

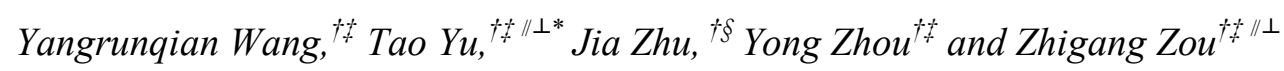

${ }^{\dagger}$ National Laboratory of Solid State Microstructures, Nanjing University, Nanjing 210093, P. R. China

†Ecomaterials and Renewable Energy Research Center (ERERC), Department of Physics, Nanjing University, Nanjing 210093, P. R. China

${ }^{\S}$ Collage of Engineering and Applied Science, Nanjing University, Nanjing 210093, P. R. China

"Collaborative Innovation Center of Advanced Microstructures, Nanjing University, Nanjing 210093, P. R. China

${ }^{\perp}$ Jiangsu Key Laboratory for Nano Technology, Nanjing 210093, P. R. China

*Address correspondence to (C. Bao) bcxiong@126.com, (T. Yu) yutao@nju.edu.cn 


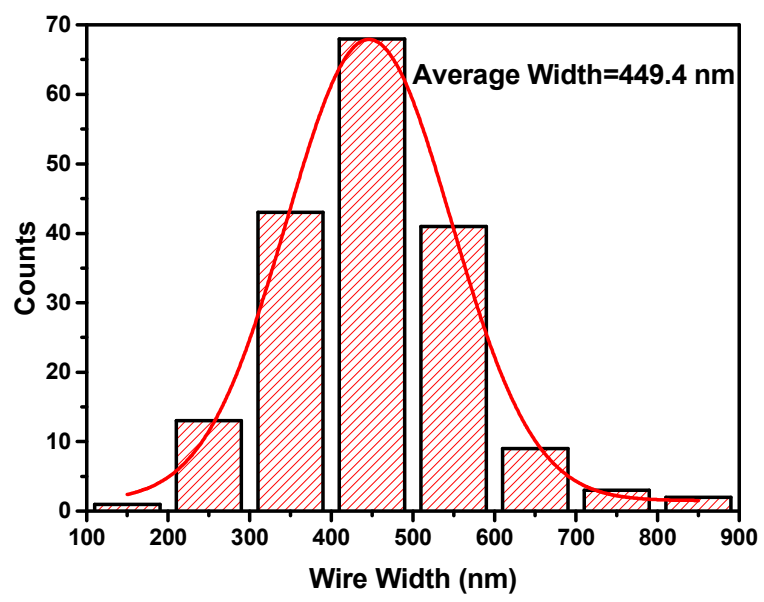

Figure S1 The width distribution of Au nanowires.

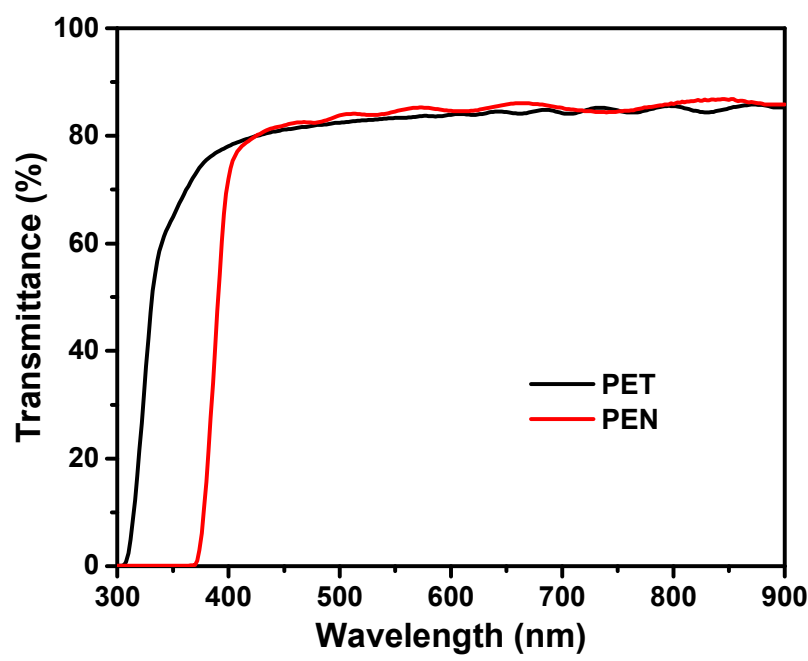

Figure S2 Transmittance spectra of PET and PEN substrates.

Table S1 Device yield rate of $\mathrm{CH}_{3} \mathrm{NH}_{3} \mathrm{PbI}_{3} \mathrm{PD}$ based on AuNW TEs.

\begin{tabular}{|l|l|l|l|}
\hline Thickness of AuNW & Sum of PDs & Sum of worked PDs & Yield rate \\
\hline $50 \mathrm{~nm}$ & 36 & 35 & $97 \%$ \\
\hline $100 \mathrm{~nm}$ & 24 & 0 & $0 \%$ \\
\hline
\end{tabular}

Among 36 devices based on $50 \mathrm{~nm}$ thick AuNW $\left(\mathrm{R}_{\mathrm{q}} \sim 20 \mathrm{~nm}\right), 35$ devices showed response to the indoor diffuse light and output an open circuit voltage $\left(\mathrm{V}_{\mathrm{OC}}\right)$ greater than $0.2 \mathrm{~V}$. While no device from 24 devices based on $100 \mathrm{~nm}$ thick AuNW $\left(\mathrm{R}_{\mathrm{q}} \sim 40\right.$ $\mathrm{nm})$ showed response to light and no $\mathrm{V}_{\mathrm{OC}}$ can be measured. 

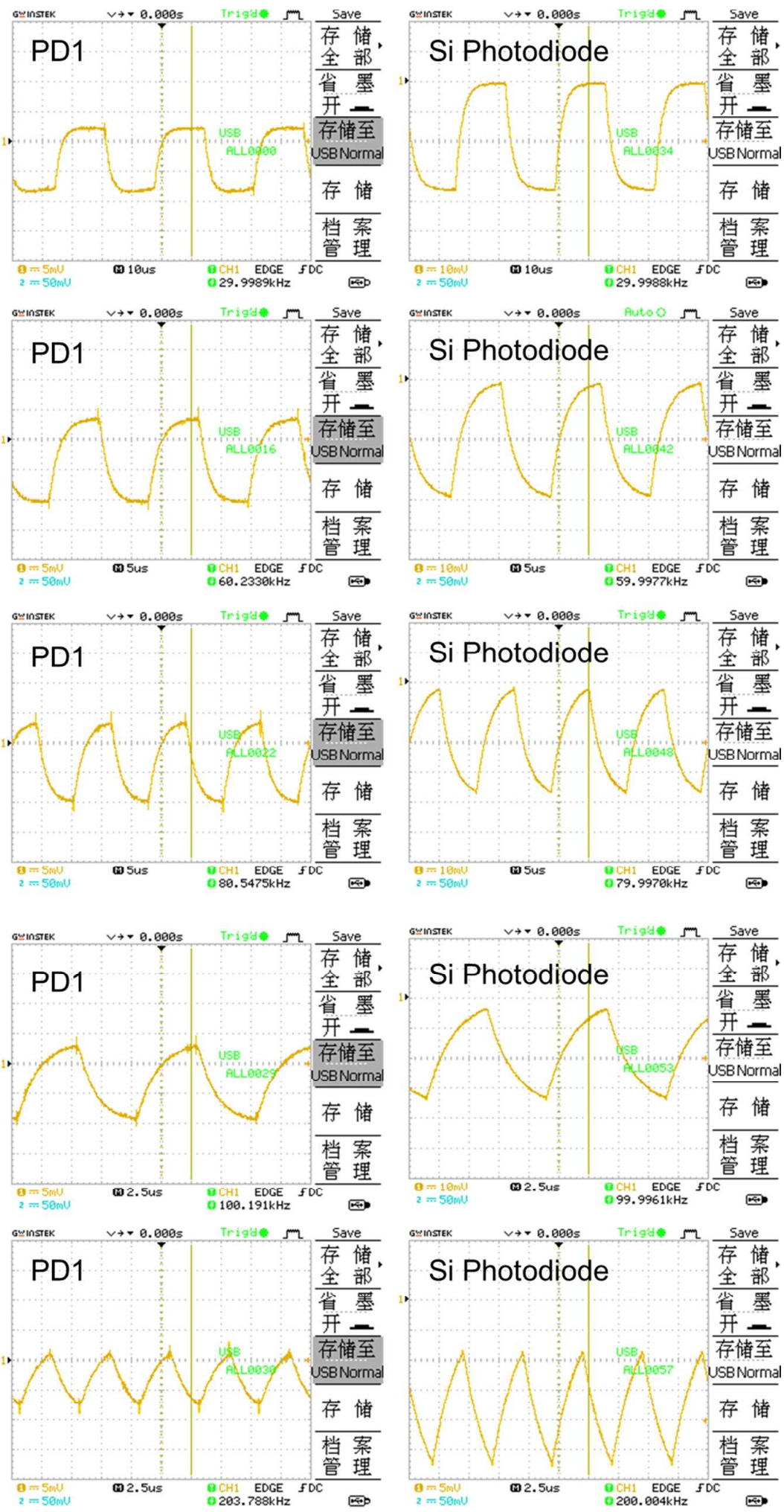

Figure S3 Transient response at a pulse frequency of $30-200 \mathrm{kHz}$ of a PEN/AuNW based flexible perovskite photodetector (PD1) with a device area of $0.09 \mathrm{~cm}^{2}$ and a Si photodiode with a device area of $0.25 \mathrm{~cm}^{2}$. 

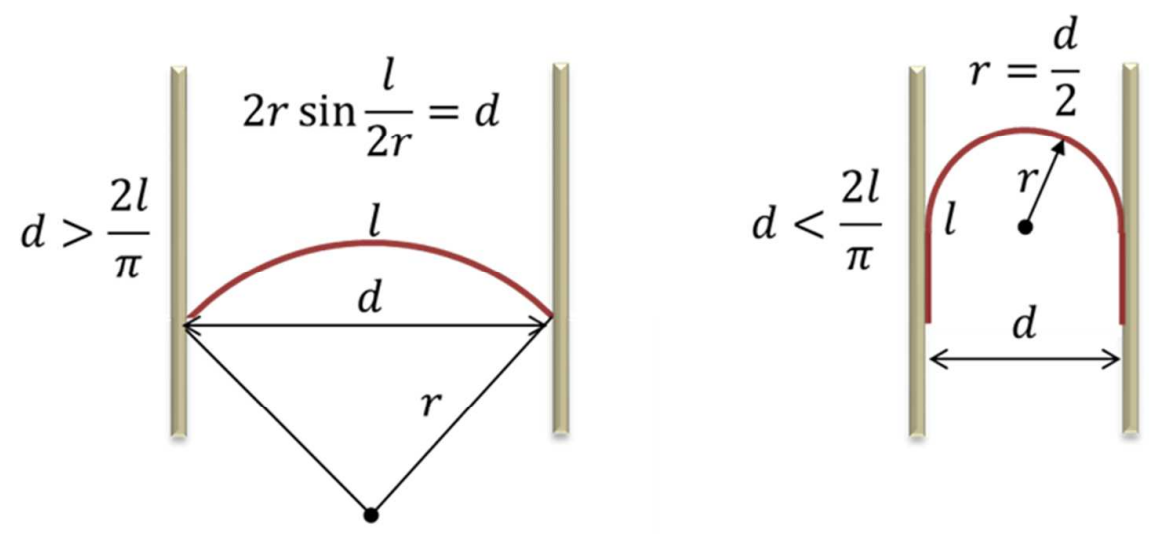

Figure S4 The relationship between the bending radius (r) and the jaws width (d).

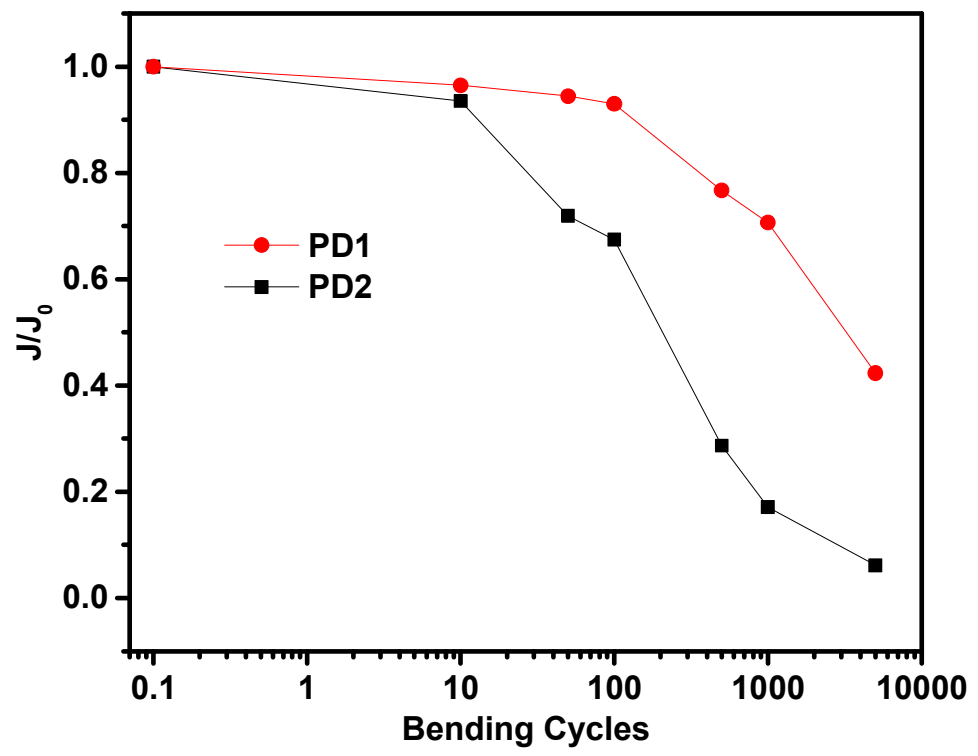

Figure S5 Photocurrent attenuation of flexible $\mathrm{MAPbI}_{3}$ photodetectors based on PEN/AuNW (PD1) and PET/ITO (PD2) transparent electrodes at $0 \mathrm{~V}$ bias as a function of bending cycles at a radius of $5 \mathrm{~mm}$. The photocurrents were measured when the bending was released. 